\title{
Asteroid-meteorite links: the Vesta conundrum(s)
}

\author{
C. M. Pieters ${ }^{1}$, R. P. Binzel ${ }^{2}$, D. Bogard ${ }^{3}$, T. Hiroi ${ }^{1}$, \\ D.W. Mittlefehldt ${ }^{3}$, L. Nyquist ${ }^{3}$, A. Rivkin ${ }^{4}$ and H. Takeda ${ }^{5}$ \\ ${ }^{1}$ Department of Geological Sciences, Brown University, Providence, RI 02912 USA \\ ${ }^{2}$ Department of Earth, Atmosphere, Planetary Science, MIT, Cambridge, Massachusetts, USA \\ ${ }^{3}$ NASA Johnson Space Center, Houston, TX 77058 USA \\ ${ }^{4}$ The Johns Hopkins University Applied Physics Laboratory, Laurel, Maryland, USA \\ ${ }^{5}$ Chiba Institute of Technology, Tsudanuma, Narashino City, Chiba 275, Japan
}

\begin{abstract}
Although a direct link between the HED meteorites and the asteroid 4 Vesta is generally acknowledged, several issues continue to be actively examined that tie Vesta to early processes in the solar system. Vesta is no longer the only basaltic asteroid in the Main belt. In addition to the Vestoids of the Vesta family, the small asteroid Magnya is basaltic but appears to be unrelated to Vesta. Similarly, diversity now identified in the collection of basaltic meteorites requires more than one basaltic parent body, consistent with the abundance of differentiated parent bodies implied by iron meteorites. The timing of the formation of the Vestoids (and presumably the large crater at the south pole of Vesta) is unresolved. Peaks in Ar-Ar dates of eucrites suggest this impact event could be related to a possible late heavy bombardment at least 3.5 Gyr ago. On the other hand, the optically fresh appearance of both Vesta and the Vestoids requires either a relatively recent resurfacing event or that their surfaces do not weather in the same manner thought to occur on other asteroids such as the ordinary chondrite parent body. Diversity across the surface of Vesta has been observed with HST and there are hints of compositional variations (possibly involving minor olivine) in near-infrared spectra.
\end{abstract}

Keywords.

\section{Introduction}

The majority of meteorites are believed to be derived from parent bodies in the asteroid belt, even though there are few specific asteroids identified as sources. A principal exception is the association of Vesta with the Howardite, Eucrite, Diogenite class of achondrites (HEDs). Shown in Figure 1 is the original data used in 1970 to argue that the mineralogical properties of the HED meteorites (largely low-Ca pyroxene + plagioclase) are the same as those observed for the asteroid Vesta (McCord et al. 1970). At that time, and for several decades later, Vesta was the only asteroid identified to have a basaltic surface. Vesta was therefore believed to be the only large asteroid remaining intact that had melted and differentiated during the early phases of solar system evolution.

Excellent reviews of the character and issues associated with the HED class of meteorites can be found in Takeda (1997), Mittlefehldt et al. (1998), Drake (2001), and Keil (2002). Even though the link to Vesta has been scrutinized for decades, a number of rather substantial new issues are actively being discussed as a result of a growing body of new telescopic measurements and detailed analyses of meteorites. A composite spectrum for Vesta is shown in Figure 2 derived from recent surveys. Several key issues are highlighted here in the context of measured properties of Vesta and the HEDs. The 


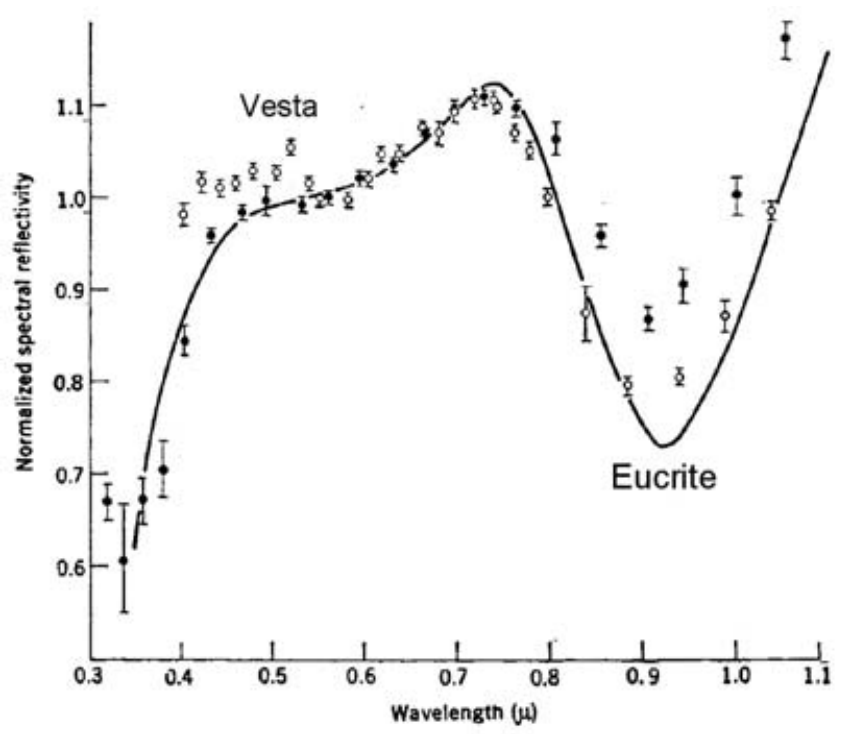

Figure 1. Original telescopic data linking Vesta and the basaltic achondrites. (after McCord et al. 1970). The absorption feature near $0.92 \mu \mathrm{m}$ for Vesta (points) is an indication of low-Ca pyroxene and is comparable to that seen in laboratory spectra of basaltic achondrites.

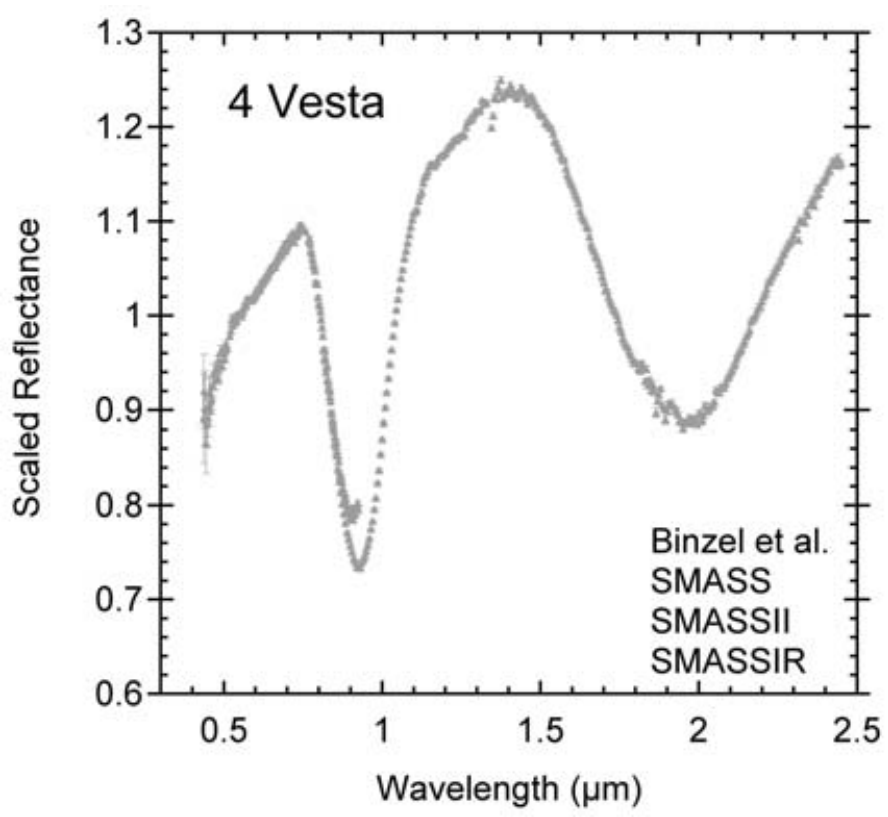

Figure 2. Composite average spectrum of Vesta derived from the SMASS programs of Binzel et al. [see: www.smass.mit.edu].

intent is to stimulate discussion that will lead to specific observations and analyses that can be addressed with data acquired during the Dawn rendezvous with Vesta.

Dawn, the 9th mission in NASA's highly successful Discovery Program, will undertake a detailed study of the geophysics, mineralogy, and geochemistry for two of the largest main belt asteroids (Russell et al. 2004). Dawn is scheduled to be launched in 2006 and 


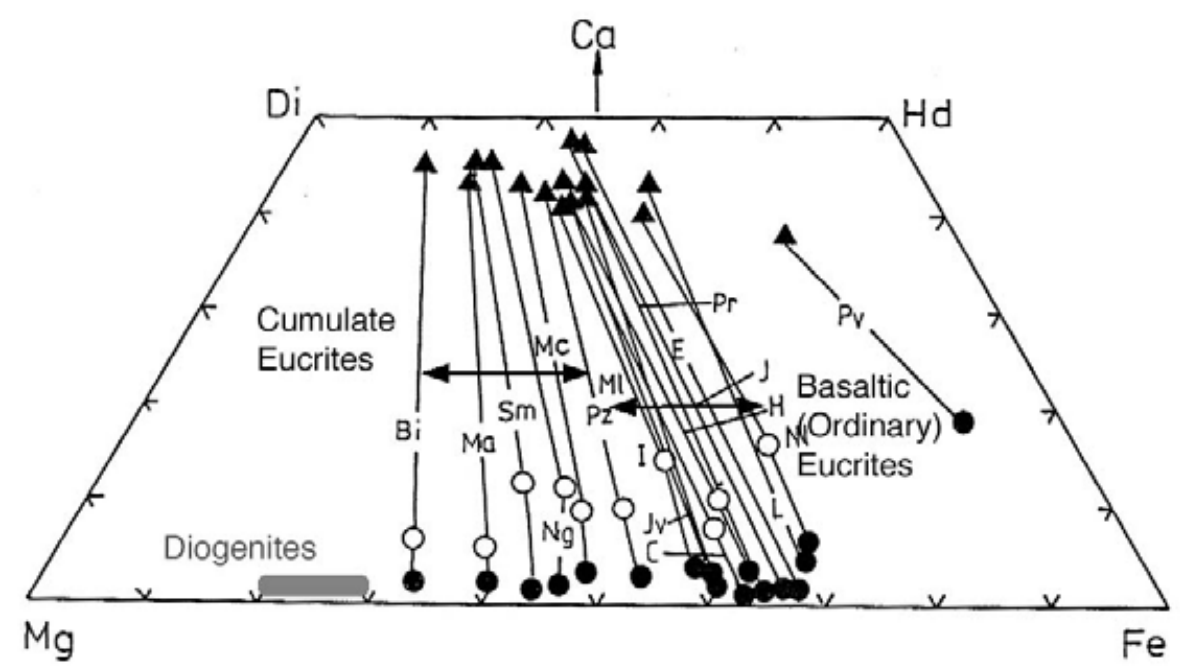

Figure 3. Pyroxene compositions for non-Antarctic Diogenites and Eucrites (after Takeda 1997). The lines connect host pyroxene and exsolution lamellae and the bulk composition is shown as open circles.

will rendezvous with Vesta in 2011 and with Ceres in 2016. The spacecraft will operate for an extended period in nested orbits at each of these minor planets. Dawn science payload includes framing cameras with multi-spectral and stereo capability, a visible to near-infrared imaging spectrometer, a gamma ray/neutron spectrometer, and radio science.

\section{The Vesta-HED Link: Is Vesta the Only Basaltic Parent-Body in the Asteroid Belt?}

The Vesta-HED link is directly tied to the observed/inferred mineralogy of the asteroid and the measured mineralogy of the meteorites. Pyroxenes are the dominant mafic mineral present in HED meteorites and provide multiple clues about how the parent body evolved (e.g., Takeda 1997). The primary distinctions of pyroxene compositions observed in non-Antarctic HEDs are summarized in Figure 3. For those monomict samples that have not been extensively reprocessed, there appears to be a continuous pyroxene compositional trends across the HED suite (Takada et al. 1983; 1997). Diogenites have the most magnesium orthopyroxenes and ordinary (basaltic) eucrites contain the most iron-rich pyroxenes. Although cumulate eucrites are clearly linked to the other eucrites, they have a cumulate texture and their pyroxenes are intermediate in $\mathrm{Mg}$ content. Howardites are breccia mixtures of diogenites and eucrites, but individual clasts often fill gaps further illustrating a continuous relation between the components.

Fortunately, remote compositional analyses using visible to near-infrared spectroscopy are very sensitive to pyroxene composition. Ferrous iron in the asymmetric M2 pyroxene site provides highly diagnostic absorptions near 1 and $2 \mu$ that vary in wavelength with the composition and structure of the pyroxene (e.g., Burns 1993). Typical examples of reflectance spectra for Eucrites, Diogenites, and Howardites are shown in Figure 4. Diogenites with their more Mg-rich pyroxenes have absorption bands at shorter wavelengths than those of the more ferrous eucrites. Howardites, a brecciated and physical mixture, 

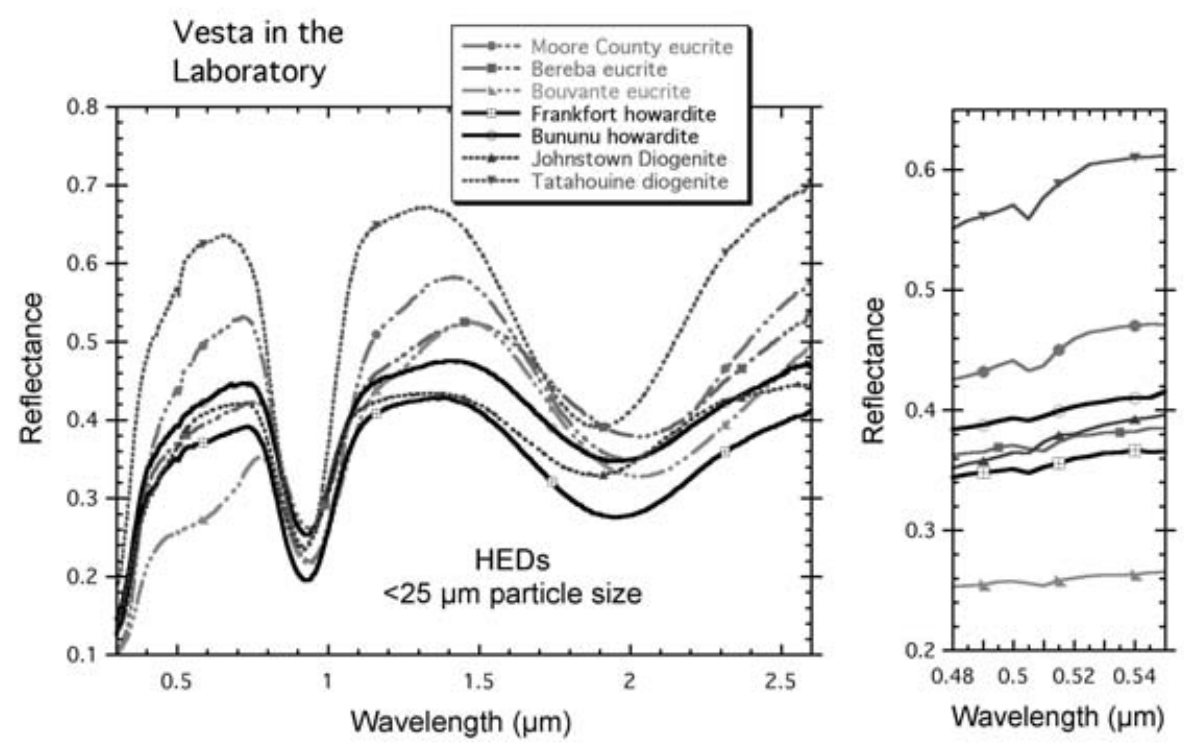

Figure 4. Laboratory bidirectional reflectance spectra of typical examples for Howardites, Eucrites, and Diogenites (HEDs). Right) The same spectra with the visible part of the spectrum expanded. All spectra were acquired at $5 \mathrm{~nm}$ spectral resolution.

exhibit spectra with composite absorption band minima intermediate between the two components of the mixture.

The spectrum of each HED meteorite is dominated by the spectral properties of pyroxene. This is illustrated with spectra of mineral separates of a cumulate eucrite, Y-980318, shown in Figure 5. The spectrum of the bulk sample is dominated by the optical properties of the pyroxene. All of the major absorptions are due to electronic transitions of ferrous iron in the M1 and M2 sites of pyroxene (Burns 1993). Ferrous iron in the M1 site of pyroxene produces weaker features than when in the M2 site because the M1 site is more symmetric. Most of the weaker features at shorter wavelengths in the visible are associated with either spin forbidden or charge transfer transitions. On the other hand, iron-poor plagioclase is not absorbing in the near infrared and does not contribute features to the bulk spectrum. The presence of plagioclase provides a relatively transparent medium and enhances the optical path length. A photomicrograph of Y-98318 is shown in Figure 6 illustrating the thick exsolution lamellae characteristic of pyroxene in cumulate eucrites (Takeda 1997).

Reflectance spectra of Vesta acquired using Earth-based telescopes represent an integrated average of the side facing Earth at the time of the measurement. Since Vesta is large (equatorial diameter $\sim 550 \mathrm{~km}$ ) and bright (IRAS albedo $\sim 0.4$ ), high quality spectra are available (e.g., Gaffey 1997 and Fig. 2). In 1993 detector sensitivity had advanced sufficiently to allow Binzel \& Xu (1993) to identify a group of very small asteroids in the vicinity of Vesta that appear to have the same basaltic composition as the larger asteroid. Reflectance spectra of Vesta and representative spectra of these "Vestoids" are shown in Figure 7 and the distribution of currently identified Vestoids are shown in Figure 8. All members of this family of Vestoids are faint, of course, and statistical errors tend to be larger than for Vesta (Xu \& Binzel 1993; Burbine et al. 2002). Increasing sensitivity of 


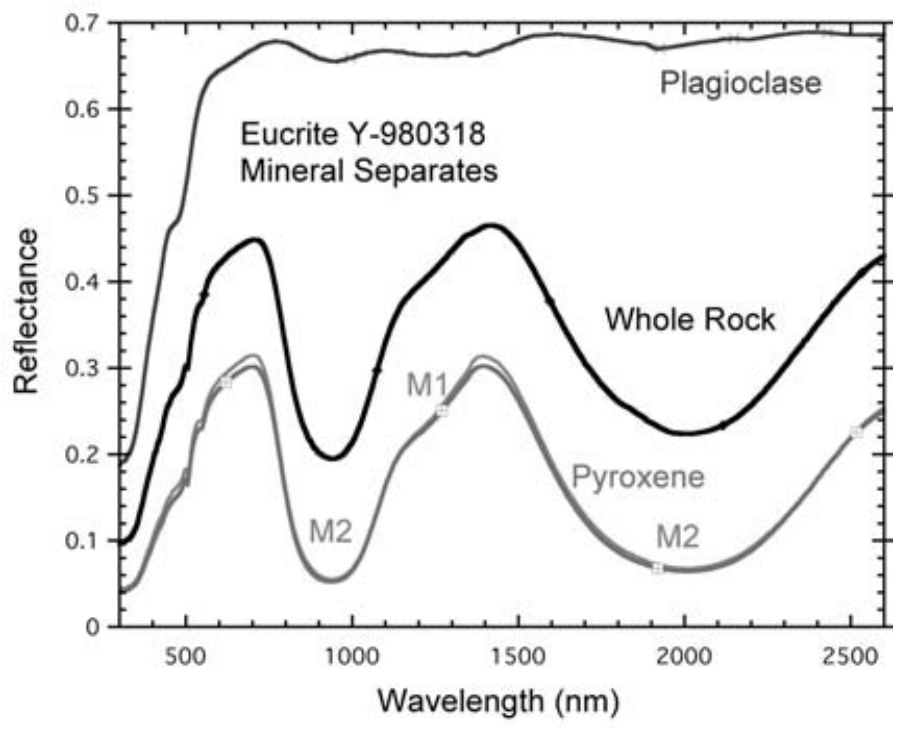

Figure 5. Bidirectional reflectance spectra for the bulk sample and mineral separates from cumulate eucrite Y-980318. The bands near 1 and $2 \mu \mathrm{m}$ are due to ferrous iron in the M2 pyroxene site, whereas the weaker band near $1.2 \mu \mathrm{m}$ is due to ferrous iron in the more symmetric M1 site of pyroxene.

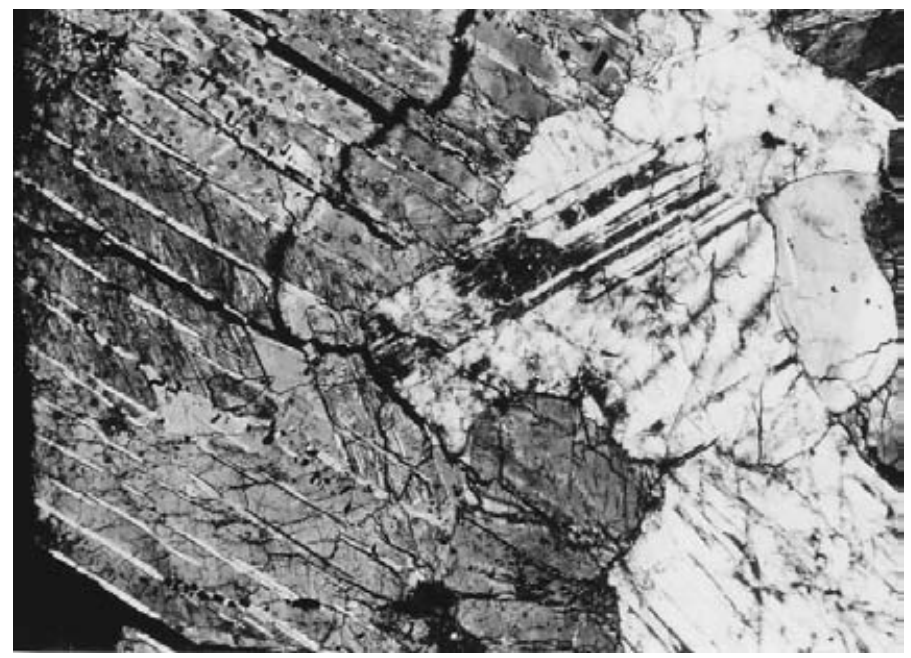

Figure 6. Photomicrograph of cumulate eucrite Y-980318 showing exsolution lamellae of orthopyroxene inverted from pigeonite. Field of view is $3.3 \mathrm{~mm}$.

modern instruments has provided data of sufficient quality to allow comparison of spectral parameters linked to mineralogy across the Vesta family (Hiroi et al. 1995, 1998; Duffard et al. 2004; Vernazza et al. 2005).

The distribution of basaltic Vestoids extends from Vesta to several key resonances known to act as "escape hatches" to the inner solar system (Wisdom 1985), and thus a mechanism was found to exist for delivery of the HED meteorites from basaltic asteroids in the main belt. This was strengthened when sequential observations with the Hubble Space Telescope allowed a three-dimensional shape model for Vesta to be developed (Thomas et al. 1997). The shape model for Vesta revealed a very large crater-like 


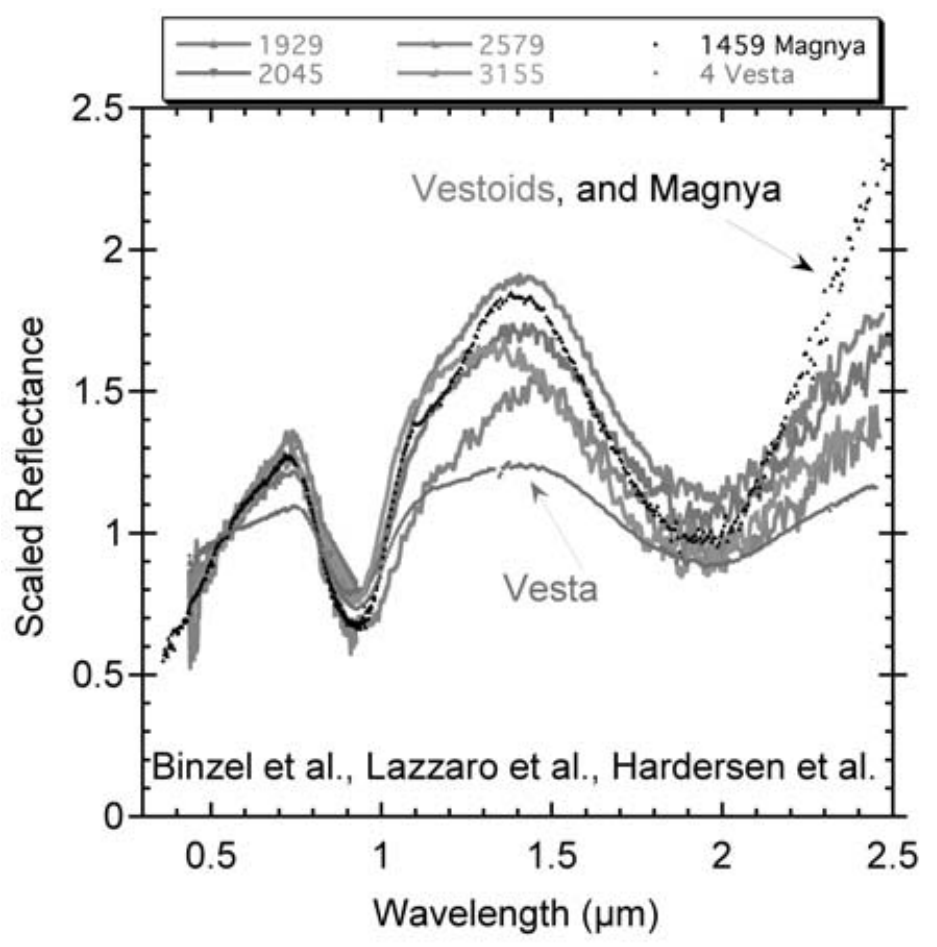

Figure 7. Telescopic reflectance spectra of Vesta, Vestoids, and Magnya. Vesta exhibits a notably flatter continuum and weaker absorption bands.

depression near the south pole as a possible excavation site for the Vestoids, and hence an ultimate source for the HEDs.

Spectra of individual HED meteorites are quite diverse and absorption features are directly linked to meteorite mineralogy (Figures $3 \& 4$ ). As might be expected for an impact mixed regolith, the principal spectral properties of Vesta's observed surface are most comparable to those of Howardites. The observed albedo and band strength, however, suggest Vesta's surface materials must also must be relatively fine grained and on the order of $<25 \mu \mathrm{m}$ (Hiroi et al. 1994). A comparison of spectra for particle size separates of howardite EET87503 is shown in Figure 9. As is typical for size separates, the finer particles are brighter and the larger particles have a flatter spectrum (larger component of first surface Fresnel reflection). The data are scaled to unity in the visible in Figure $9 \mathrm{~b}$. Vesta is seen to be most comparable to the howardite sample with a fine-grained size distribution. Since small particles always coat larger particles in a natural soil, the larger size separates actually have very little analytical use in spectral comparisons and modeling in the near-infrared.

There are a few very important observations, however, that do not readily fit into an all encompassing Vesta-HED story. As can be seen in Figure 7, the Vestoids generally have stronger absorption bands than Vesta and they exhibit a notably steeper continuum from the visible to $1.6 \mu \mathrm{m}$. Based on the wavelength of the primary pyroxene absorption bands, the first order pyroxene mineralogy of Vesta and the Vestoids is similar. Both represent basaltic rock types. However, there is no question that the uppermost surface of the large asteroid observed remotely is quite different from that of the smaller relatives (e.g., Hiroi et al. 1995, 2001). It is not known yet whether these differences are due to physical properties and/or texture differences, compositional variations implying different source 


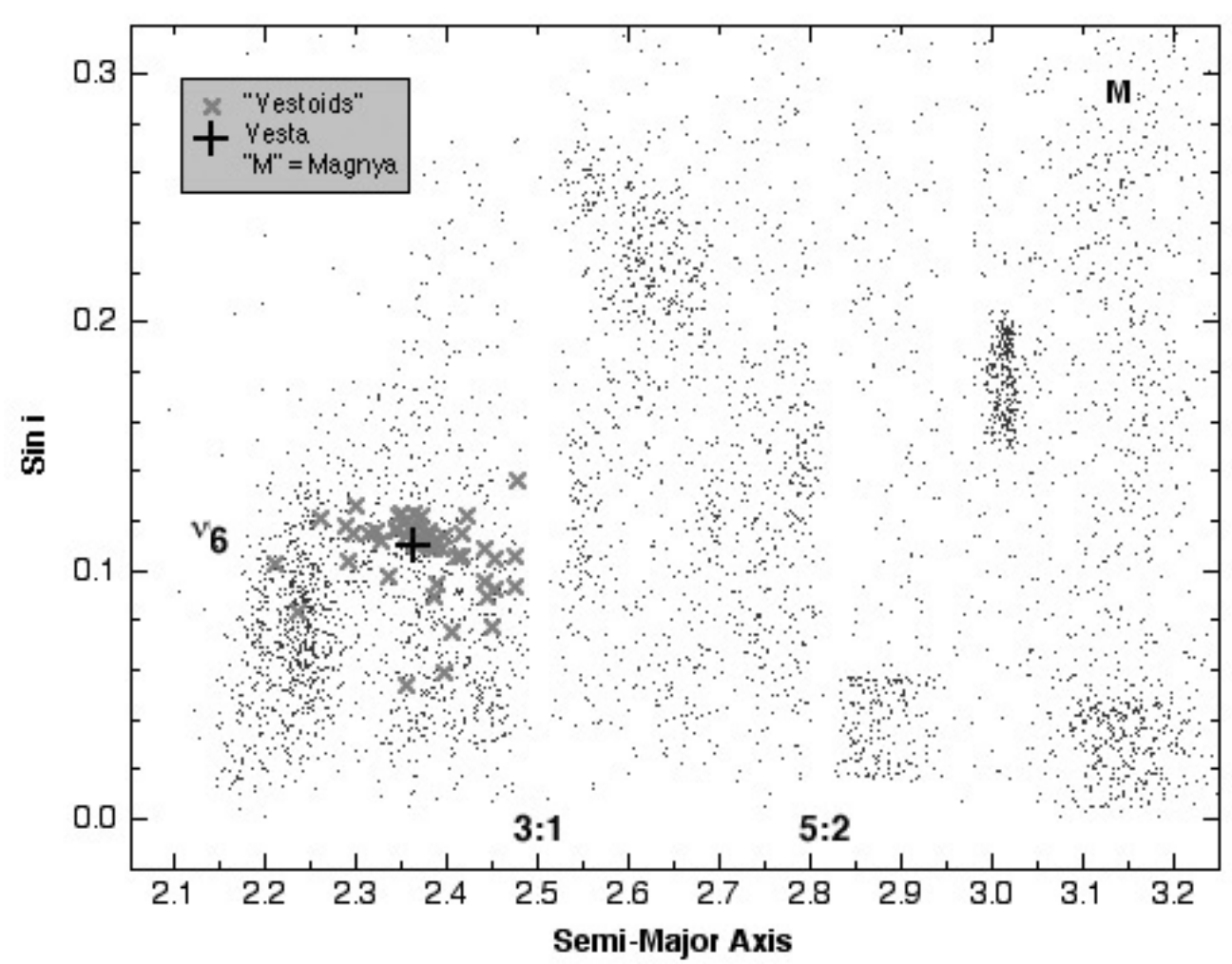

Figure 8. Orbital parameters of Vesta $(+)$, Vestoids (x), and Magnya (M) within in the main asteroid belt (dots). Principal resonances are labeled.

material, or environmental processes that alter surface materials. Examples include: a fine coating of feldspathic dust on a coherent basaltic substrate, a coarse grain basaltic fragment with relatively Fe-rich pyroxenes, a gravity dependent space weathering process, etc. A separate parent body for the Vestoids is not needed, but cannot be eliminated with the current data.

As the character and diversity of small bodies continue to be explored with increasingly sensitive instruments, additional exceptions are found. A small ( $\sim 30 \mathrm{~km}$ diameter) basaltic asteroid, Magnya, was identified in the outer asteroid belt far removed from Vesta (Lazzaro et al. 2000), on the other side of several major resonances (see Figure 8). The pyroxene bands for Magnya are at a shorter wavelength than those of Vesta (Hardersen et al. 2004) which implies a more $\mathrm{Mg}$ bulk pyroxene composition. Independent of actual magnitude, the albedo of Vesta appears to be about twice as high as that of the much smaller Magnya (Hardersen et al. 2004). Such a difference could be due to bulk composition, but is more likely a clue to inherent textural differences of small basaltic asteroid fragments. Magnya itself is likely to be a small remnant of a larger body distinct from Vesta. From a dynamical point of view, it is very unlikely that Vesta and Magnya can be related (Lazzaro et al. 2000). Thus, Magnya is currently the best evidence for a possible second basaltic parent body having existed in the main asteroid belt. 


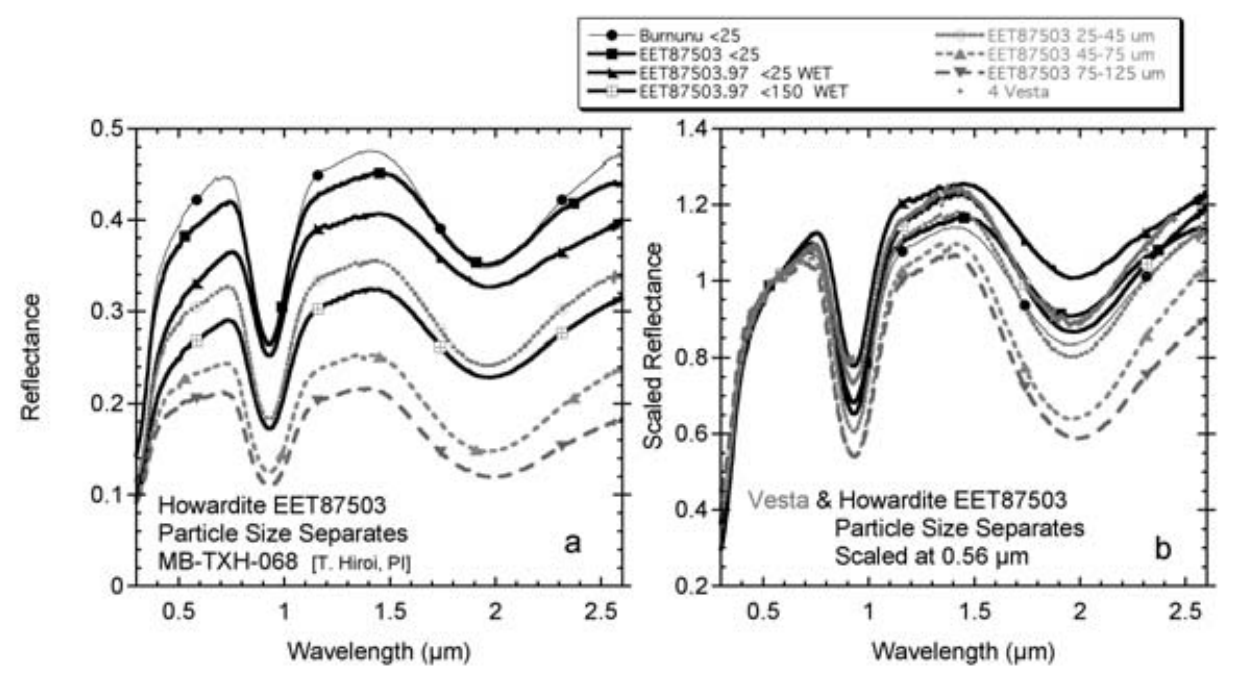

Figure 9. a) Laboratory bidirectional reflectance spectra for bulk powders (lines) and particle size separates (dash, dots) of Howardite EET87503. A spectrum of howardite Bununu is shown for comparison. b) The same as (a), but scaled to unity at $0.56 \mu \mathrm{m}$. The composite spectrum of Vesta from Figure 2 is included for comparison in (b). Note that the optical characteristics of the $45-75$ and $75-125 \mu \mathrm{m}$ separates are all outside the range of the $<150$ bulk properties even though their actual size is $<150 \mu \mathrm{m}$.

\section{The Vesta-HED Link: Do All Basaltic Meteorites Share the Same Parent Body?}

Oxygen isotopes have long been used to group meteorite classes (Clayton et al. 1993) and distinct fields of oxygen isotope values have been interpreted to imply the evolution of different parent bodies. The general presumption is that large differences in oxygen isotopic values implies very different parts of the solar system. The HED meteorites form their own group, another piece of evidence arguing that they have a common parent body, Vesta.

Instrumentation has also improved in terrestrial laboratories and more detailed and higher precision analyses are now possible for small valuable meteorite samples. Two basaltic meteorites classified as eucrites have recently been shown to have oxygen isotopic signatures significantly different from the main family of HEDs: NWA011, which falls far from other HEDs and closer to the CR-chondrites (Yamaguchi et al. 2002; Floss et al. 2005), and Ibitira which falls between the HED and terrestrial fractionation line (Wiechert et al. 2004). Distinction of Ibitira is only made possible with the high precision that can be achieved with modern instruments (Wiechert et al. 2004). There are several additional geochemical parameters that suggest the parent body of Ibitira is distinct (Mittlefehldt 2005). A composite figure illustrating the oxygen isotope relations for HEDs, NWA011, and Ibitira is shown in Figure 10.

Minor elements such as $\mathrm{Ti}, \mathrm{Cr}$, and $\mathrm{Al}$ in the pyroxene structure are known to significantly affect the character of absorption bands as well as the nature of the continuum. However, no systematic analysis documenting the spectroscopic effects of such elements in the pyroxene structure has been undertaken for pyroxenes in the HED range of compositions. Until such effects are better defined, spectra of the two unique basaltic meteorites, Ibitira and NWA011, cannot be readily used to identify their parent body. Both are 


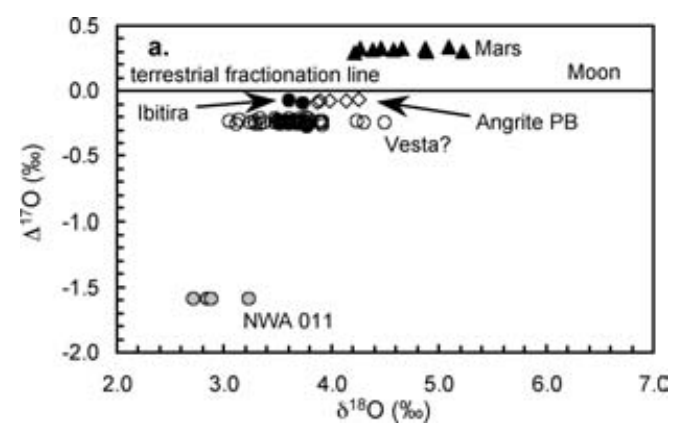

(a)

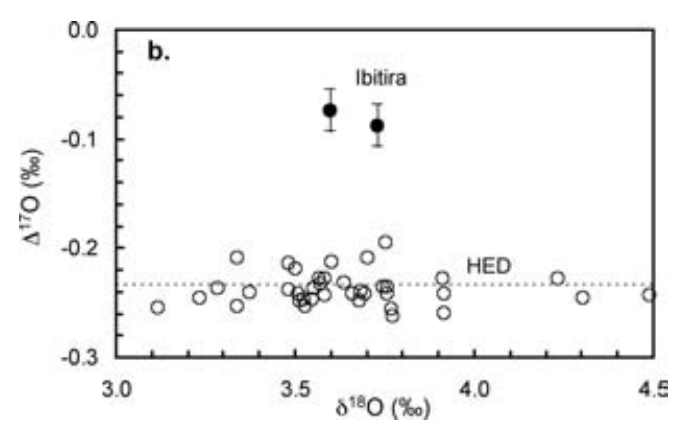

(b)

Figure 10. Recent oxygen isotope measurements for the inner planets, HEDs, and other basaltic meteorites. (data taken from Floss et al. 2005; Franchi et al. 1999; Greenwood et al. 2005; Wiechert et al. 2001, 2004). Figure (b) contains the same data as (a) at an expanded scale.
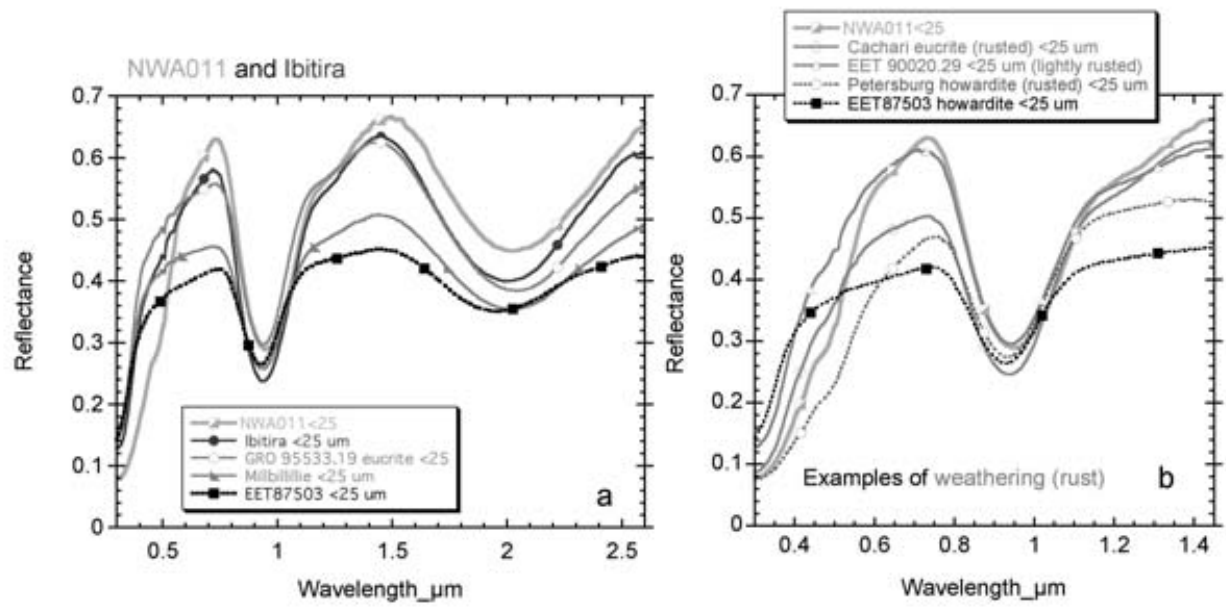

Figure 11. Bidirectional reflectance spectra for NWA011, Ibitira and similar examples of HEDs. All exhibit diagnostic properties indicative of basaltic composition.

basaltic, but neither exhibit spectra that can be distinguished from the diverse range of HEDs measured (see the collection of Takahiro Hiroi [TXH] in the RELAB database at http://www.planetary.brown.edu/relab. Shown in Figure 11 are reflectance spectra of these two unusual meteorites along with examples of eucrites with similar bulk pyroxene composition. NWA011 (sample provided by Akira Yamaguchi) has the unfortunate additional characteristic of terrestrial weathering. Examples of the effects of terrestrial weathering is easily seen in the visible part of the spectrum for NWA011.

\section{When Did Major Geological Events Occur on Vesta?}

A detailed discussion of the timing and character of the original heating event that produced the basaltic character of Vesta and the HEDs is beyond the scope of this overview. Differentiation occurred only a few million years after the formation of the CAIs and chondrules. As an example, a summary of the early closure dates for cumulate 


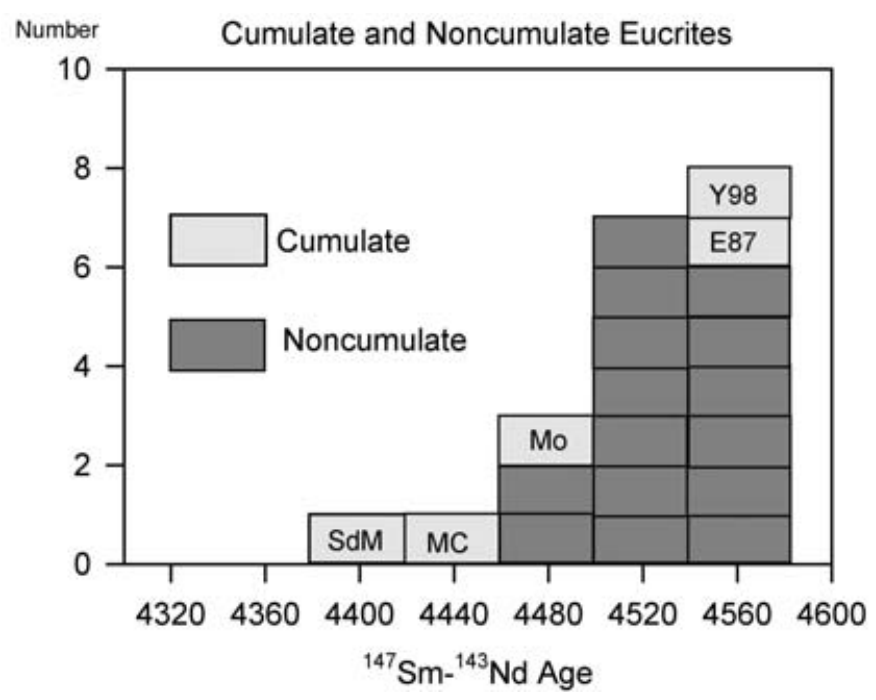

Figure 12. Summary of Sm-Nd ages for cumulate and non-cumulate eucrites [after Nyquist 2005]. The cumulate eurcrites (blue) are labeled: Serra de Mage (SdM), Moore County (MC), Moama (Mo), Yamato 980318, and EET87520. Data from Carlson \& Lugmair (2000; EET87520, Moama, Moore County, Serra de Mage) and Nyquist et al. (2004; Y980318).

and non-cumulate eucrites as measured by Sm-Nd is shown in Figure 12 (Nyquist 2004). These ancient dates set limits for when Vesta had a solid crust composed of materials that we recognize as HED meteorites.

Discussion of the character of a magma ocean and the relative influence of partial melting and fractional crystallization in the formation of the different types of eucrites and diogenites continues within the meteorite community. The debate focuses largely on whether partial melting or fractional crystallization is more important for the regular trends of compositions observed in the HEDs. Regardless how the components were formed, the resulting sequence is generally believed to be arranged similar to the layered crust model of Takeda $(1983,1997)$ which is illustrated in Figure 13. Impact craters reworked the surface during and after formation of the primary zones, rearranging and mixing components on a scale that depends on the size of the impact and the depth of the compositional zones. Deposits from the largest, and perhaps earliest, craters also provided sufficient thermal environment for moderate metamorphism.

Argon isotopic analyses are known to be disturbed or reset by major impact events and Ar-Ar dating is thus a good method to track some of these early events (Bogard 1995). Summarized in Figure 14 are Ar-Ar ages for eucrites presented as probability plots (after Bogard 2005). The clustering of data between 4 and $3.5 \mathrm{Gyr}$ is highly suggestive of a period of heavy bombardment similar to the late heavy bombardment suggested for the Moon from lunar samples (Ryder et al. 2002). The magnitude of these impacts on the HED parent body (Vesta) would be consistent with energy required to have launched the Vestoids. Since the HEDs seem to share several common peaks in Figure 14, the youngest peak at 3.5 Gyr may indicate that samples destined to become HEDs resided on Vesta until at least as recently as 3.5 Gyr. From a collisional point of view, younger ages for the formation of Vestoids.become increasingly less likely because of the rarity of giant impacts sufficient to form the south pole basin and excavate the Vestoids.

Whenever the Vestoids were formed, the impact event must have been very large and scattered fragmental debris across Vesta itself as well as dusted the surface of the 


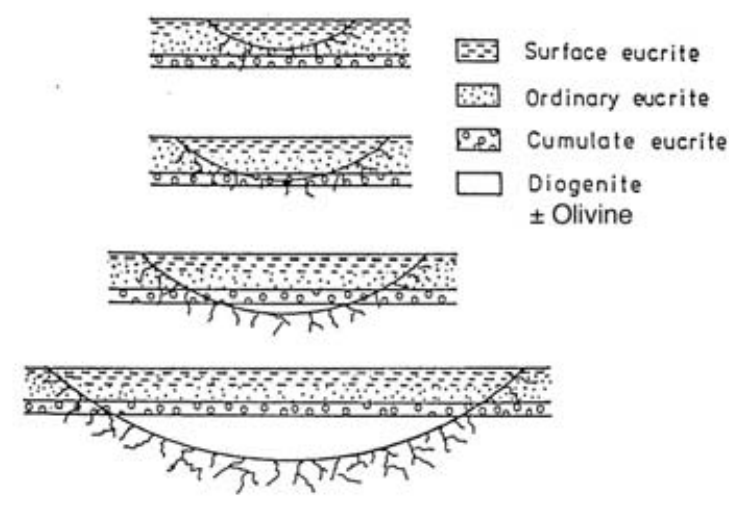

(a)

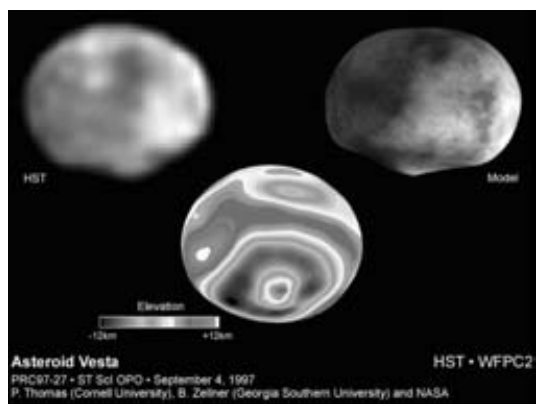

(b)

Figure 13. Layered crust model for Vesta (after Takeda 1997). Impact craters excavate (and mix) different compositional zones. Large craters would produce mixed compositions like the Howardites. The largest crater, such as that observed by HST near Vesta's south pole shown on the right (after Thomas et al. 1997), may have formed and distributed the Vestoids.

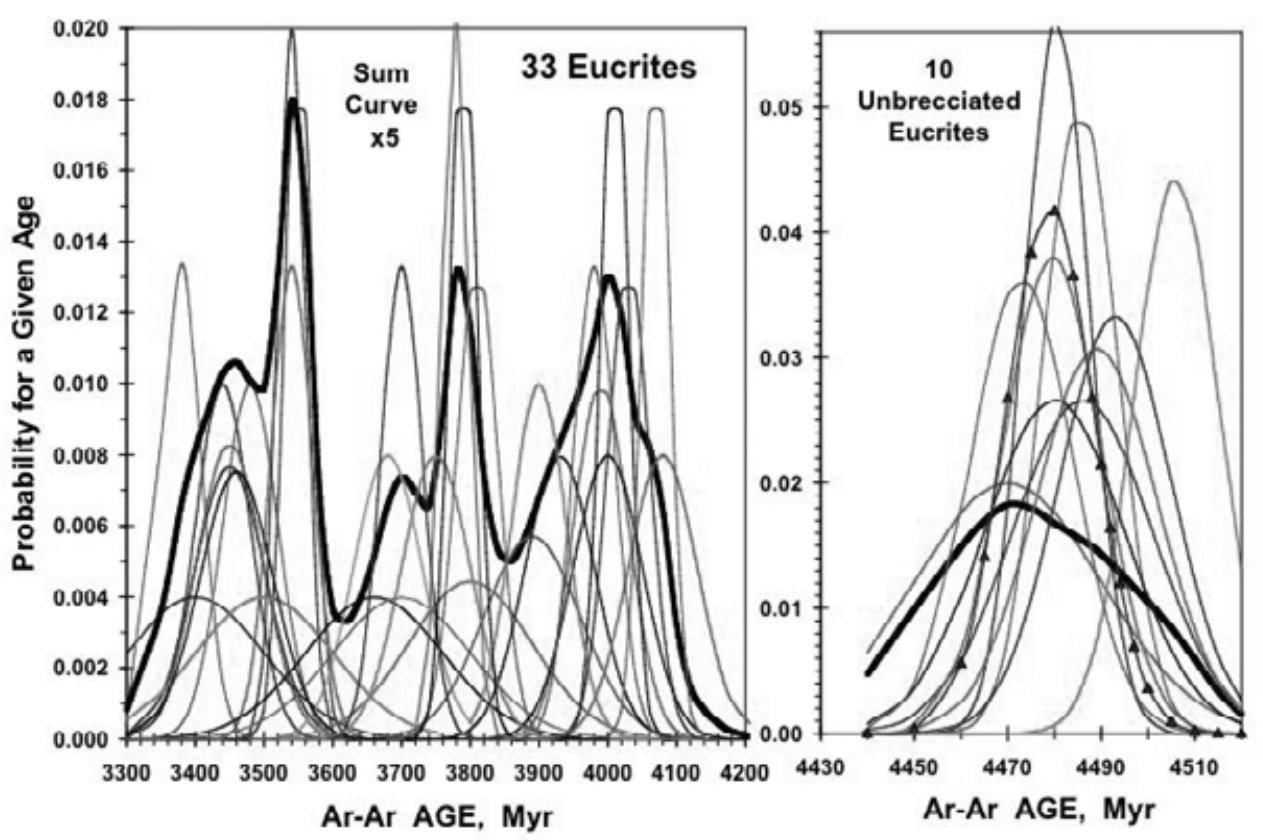

Figure 14. Combined Ar-Ar ages of eucrites (after Bogard, 2005). The probability plot on the left gives impact reset ages of brecciated eucrites. The probability plot on the right gives ages of non-brecciated (basaltic and cumulate) eucrites. The age axes of the two plots are approximately continuous, but they are different in scale. The age peak for the unbrecciated Eucrites is most prominent. The data indicate age resetting events at 4.48, 4.0, 3.8, and $3.5 \mathrm{Gyr}$. 
Vestoids. Both Vesta and the Vestoids exhibit strong diagnostic absorption bands that are quite comparable in wavelength, shape and strength to similar bands found in HED meteorites. This link to the HEDs implies that the surface of Vesta and the Vestoids are directly comparable to particulate samples of HED meteorites and have experienced very little, if any, weathering in the space environment (e.g., Pieters et al. 2000).

The optically fresh appearance of the surface of both Vesta and the Vestoids leads to a dilemma, however. If the fresh appearing surface is due to a resurfacing event, it implies that the event happened recently (e.g., Pieters \& Binzel 1994). Since both Vesta and all the Vestoids share the same fresh surface property, the event that caused it may have occurred simultaneously - i.e. the formation of the Vestoids. This is inconsistent with the much older Ar-Ar ages suggested for the formation of the large south pole crater and Vestoids.

On the other hand, perhaps the surface geology of both Vesta and Vestoids are indeed as old as suggested by the Ar-Ar data, and the optically fresh appearance is due to a lack of space weathering effects for some other reason. For example, laser induced space weathering experiments indicate pyroxene-rich surfaces are less easily altered than those rich in olivine (Sasaki et al. 2002), indicating that the rate of space weathering on the HED parent body would be slower than that of a parent body for olivine-bearing ordinary chondrities. In addition, perhaps the lack of metallic iron for HED-like materials and Vestas location in the main asteroid belt combine to produce an exceptionally low rate of space weathering, and the integrated surface exposure time is insufficient to alter the optical features. Analytical measurement and calculations for the rate of space weathering in the Vesta environment are currently not well bounded.

The dynamical estimates for when the Vestoids of the Vesta family were formed are somewhat intermediate between the Ar-Ar ages and the space weathering predictions, namely $\sim 1$ Gyr (e.g. Marzari et al. 1996). It will be very important for Dawn observations of Vesta's geology and composition to resolve this issue since it relates to the nature and timing of major events in the early solar system.

\section{Compositional Variability on Vesta?}

Although there is good agreement that the general character of the surface of Vesta is comparable to the basaltic HEDs, the geologic context of specific rock types and/or range of composition is needed to constrain the geologic evolution of the surface. From HST images of Vesta in 4 spectral bands $(0.4$ to $1.0 \mu \mathrm{m})$ we know that the surface is not covered with a uniform debris, but instead exhibits local color variations of 10-20\% (Binzel et al. 1997). From earth-based telescopes only broad variations can be evaluated as the asteroid rotates. Rotational variations are subtle, but hemispheric correlated spatial differences can be detected (e.g., Gaffey 1997). Two examples provide tantalizing clues of what may be observed at higher resolution when Dawn arrives in 2011.

Water on Vesta. There is a hint that small amounts of water or aqueous fluid existed on Vesta, probably as a contaminant from elsewhere, but possibly linked to early aqueous processes. Evidence comes both from the samples (Treiman et al. 2004) as well as telescopic observations (Hasegawa et al. 2003, Rivkin et al. 2005). Observations across the $3 \mu \mathrm{m}$ water band are difficult from Earth due to telluric water in Earth's atmosphere. A summary of variations for this part of the spectrum are shown in Figure 15 as a function of rotation (after Rivkin et al. 2005). Although some spectral variations appear to be regular with rotational phase, variations due to a hydrous component are not easily distinguished from other compositional variations. 


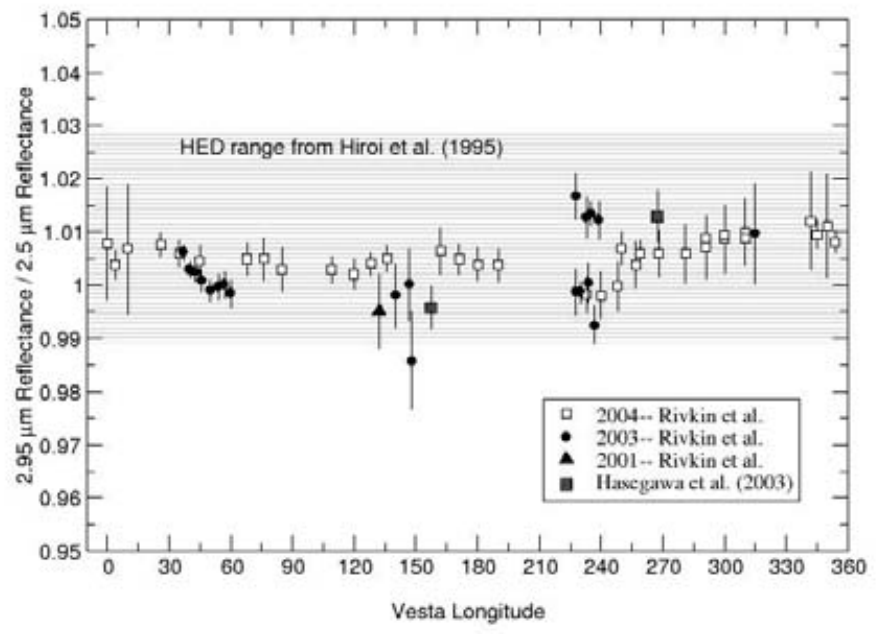

Figure 15. Rotational variations of Vesta spectra near the $3 \mathrm{~m}$ water band (after Rivkin 2005). Although the $2.95 / 2.50 \mu \mathrm{m}$ ratio should capture variations in water content, small variations in the continuum slope are of the same magnitude.

Olivine on Vesta. The size of the south pole crater on Vesta (Fig. 13) implies material excavated from several $10 \mathrm{~s}$ of $\mathrm{km}$ in the interior. Most models of a differentiated asteroid include an olivine-rich mantle, but no olivine meteorites occur in the HED suite, although olivine is a minor phase observed in some diogenite meteorites. However, one North Africa olivine diogenite meteorite has recently been found (NWA1877) that contains $45 \%$ olivine (Irvine et al. 2005). NWA1877 also contains chromite compositions that suggest a primitive mantle source.

The rotationally resolved spectra of Vesta by Gaffey (1997) are all dominated by pyroxene, but one zone on this body is subtly, but distinctly, different. As shown in Figure 16 small variation of band area ratio parameter is observed on Vesta by Gaffey. Band area ratios have been shown to be a useful tool to track mineral ratios in a mixture of olivine and orthopyroxene (Cloutis et al. 1986). The technique involves producing a ratio of the area of an absorption near $2 \mathrm{~m}$ relative to a continuum (BII) to a comparable area of the absorption near $1 \mu \mathrm{m}(\mathrm{BI})$. For low-Ca pyroxene common on Vesta (Figure 2) both of these bands are prominent. Olivine, on the other hand, contains no $2 \mu \mathrm{m}$ band and has a much broader absorption near $1 \mu \mathrm{m}$. Thus, in a mixture of orthopyroxene and olivine the band area ratio decreases with increasing olivine content. These rotational data of Vesta have characteristics consistent with a significant olivine component (Gaffey 1997). The data are tantalizing, but without additional data are unfortunately nonunique. Several groups have recently been investigating a range of parameters and find that other variables can also affect the band area ratio such as grain size, temperature, space weathering, and additional mineral diversity. Shown in Figure 17 is a summary of these effects prepared by Ueda et al. (2000). Nevertheless, there is no doubt that compositional variations exist on Vesta that are geologically significant (Binzel et al. 1997, Gaffey 1997). The instruments on Dawn (Russell et al. 2004, 2005) are well poised to characterize the surface composition in the geologic context needed. 


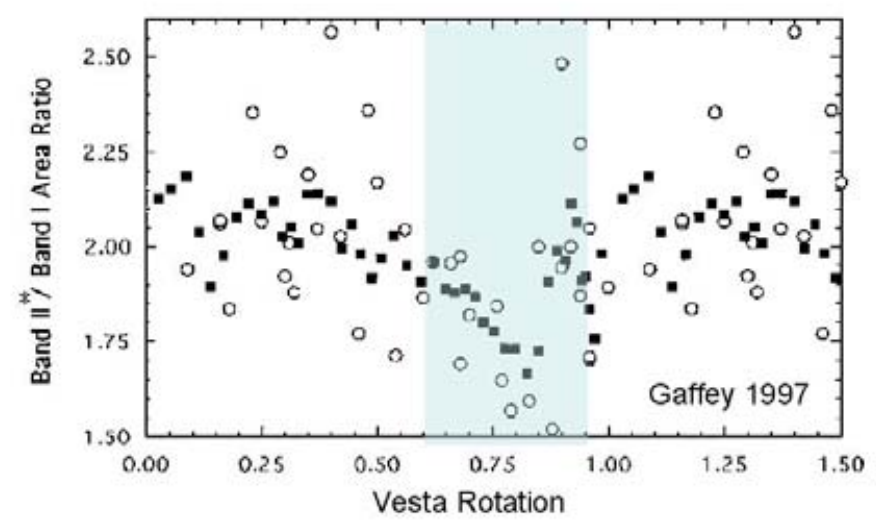

Figure 16. Relative change of the band area ratio (the $2 \mu \mathrm{m}$ pyroxene band area divided by the area of the combined ferrous band near $1 \mu \mathrm{m}$ ) as a function of rotational position of Vesta (after Gaffey 1977). The solid squares are a 5-point running average. There is one position on Vesta where the $2 \mu \mathrm{m}$ band appears relatively weaker (shaded zone near 0.75 ). This change in the relative strengths of the two bands is consistent with a variety of compositional parameters including a) a minor olivine component since olivine lacks a $2 \mu \mathrm{m}$ band, b) finer grain size, or c) variations in space weathering.

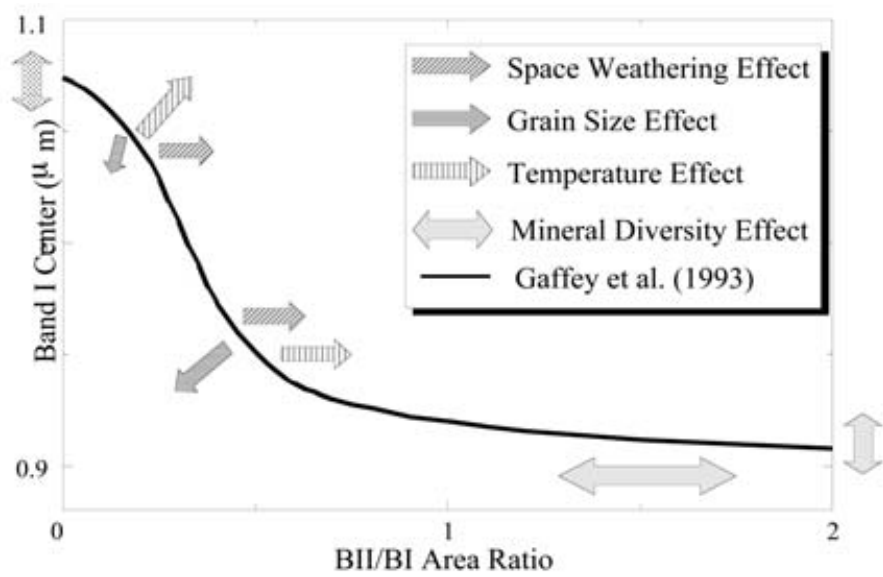

Figure 17. Schematic trends on the Band I center vs. BII/I area ratio plot of Gaffey et al. (1993) caused by space weathering effects, grain size, temperature and mineral diversity (after Ueda et al. 2000).

\section{Summary and Expectations}

There has been significant advancement to our understanding of the Vesta - HED link since the original discovery decades ago by McCord et al. (1970):

- Is Vesta the ONLY basaltic parent-body in the asteroid belt?

Vesta remains the only intact basaltic asteroid. Although both the smaller Vestoids and Magnya are clearly basaltic in nature, the Vestoids are probably related to Vesta, but Magnya is not.

- Do ALL basaltic meteorites share the same parent body? 
No. HEDs, NWA011, and Ibitira have very similar basaltic mineralogy, but appear to have distinct parent bodies. The plethora of parent bodies required by the suite of iron meteorite implies the existence of many additional differentiated parent bodies in the early solar system (e.g., Mittlefehldt et al. 1998).

- When did major geologic events occur on Vesta?

The date for the event that formed the large unnamed crater at the pole and the Vestoids is unresolved. The timing of the formation of the Vestoids is closely interwoven with understanding the space weathering environment at Vesta.

- What is the compositional range of materials on Vesta?

The layered crust model for ordinary eucrites - cumulate eucrites - diogenites (top to interior) can be expanded to include olivine diogenites. Although the currently observed surface is dominated by mixtures (howardites), spatial variations are known to exist across Vesta but their inherent compositional distinctions are ambiguous.

When high spatial resolution compositional data for Vesta is acquired by Dawn along with topography and shape, the geologic context will allow many of these issues concerning early evolution of silicate bodies in the solar system to be addressed and resolved. In the process, we will also inevitably find many many more questions about the character and history of small bodies in the solar system that we have not yet even imagined.

\section{Acknowledgements}

We thank several additional scientists who generously provided data, insights, or encouragement for this discussion: P. Buchanan, A. Coradini, M. C. De Sanctis, M. Drake, P. Hardersen, T. McCord, T. McCoy, L. McFadden, H. McSween, and C. Russell.

\section{References}

Binzel, R.P. \& Xu, S. 1993, Science 260, 186

Binzel, R.P., Gaffey, M.J., Thomas, P.C., Zellner, B.H., Storrs, A.D., \& Wells, E.N. 1997, Icarus 128,95

Binzel, R.P., Bus S.J., \& Burbine, T.H. 1999, Lunar. Planet Sci. XXX \#1216

Bogard, D.D. 1995, Meteoritics 30, 244

Bogard, D.D. \& Garrison, D.H. 2003, Meteoritics \& Planetary Science 38 (5), 669

Bogard, D.D. 2005, Lunar \& Planetary Science Conference XXXVI \#1131

Burbine, T.H., Buchanan, P.C., Binzel, R.P., Bus, S.J., Hiroi, T., Hinrichs, J.L., Meibom, A., \& McCoy, T.J. 2001, Meteoritics $\&$ Planetary Science 36, 761

Burns, R.G. 1993, Mineralogical Application of Crystal Field Theory (Cambridge: Cambridge University Press) p. 551

Carlson, R.W. \& Lugmair, G.W. 2000, in: R.M. Canup \& K. Righter (eds.), Origin of the Earth Es Moon (Tucson: University of Arizona Press), p. 25

Clayton, R.N. 1993, Annu. Rev. Earth Planet. Sci. 21, 115

Cloutis, E.A., Gaffey, M.J., Jackowski, T.L., \& Reed, K.L. 1986, Journal Geophysical Research 91, 11, 641

Cochran, A.L. \& Vilas, F. 1998, Icarus 134, 207

Cochran, A.L, Vilas, F., Jarvis, K.S., \& Kelley, M.S. 2004, Icarus 167, 360

Drake, M.J. 2001, Meteoritics 83 Planetary Science 36, 501

Duffard, R., Lazzaro, D., Licandro, J., De Sanctis, M.C., Capria, M.T., \& Carvano, J.M. 2004, Icarus 171,120

Floss, C., Taylor, L.A., Promprated, P., \& Rumble III, D. 2005, Meteoritics E Planetary Science 40,343

Franchi, I.A., Wright, I.P., Sexton, A.S., \& Pillinger, C.T. 1999, Meteoritics \& Planetary Science 34,657

Gaffey, M.J. 1977, Icarus 127, 130 
Gaffey, M.J., Bell, III, J.F., Brown, R.H., Burbine, T.H., Piatek, J.L., Reed, K.L., \& Chaky, D.A. 1993, Icarus 106, 573

Greenwood, R.C., Franchi, I.A., Jambon, A., \& Buchanan, P.C. 2005, Nature 435, 916

Hasegawa, S., Murakawa, K., Ishiguro, M., Nonaka, Hi., Takato, N., Davis, C.J., Ueno, M., \& Hiroi, T. 2003, Geophys. Res. Let. 30 (21), 2123

Hardersen, P.S., Gaffey, M.J., \& Abell, P.A. 2004, Icarus 167, 170

Hiroi, T., Pieters, C.M., \& Takeda, H. 1994, Meteoritics 29, 394

Hiroi, T., Binzel, R.P., Sunshine, J.M., Pieters, C.M., \& Takeda, H. 1995, Icarus 115, 374

Hiroi, T. \& Pieters, C.M. 1998, in: T. Harasawa (ed.), Antarctic Meteorite Research (Tokyo: National Institute of Polar Research) p. 163

Hiroi, T., Pieters, C.M., Vilas, F., Sasaki, S., Hamabe, Y., \& Kurahashi, E. 2001, Earth Planets Space 53, 1071

Irving, A.J., Kuehner, S.M., Carlson, R.W., Rumble, D. III, Hupé, A.C., \& Hupé, G.M. 2005, Lunar. Planet Sci. XXXVI \#2188

Keil, K. 2002, in: W.F. Bottke, A. Cellino, P. Paolicchi \& R.P.Binzel (eds.), Asteroids III (Tucson: University Arizona Press), p. 573

Lazzaro, D., Michtchenko, T.A., Carvano, J.M., Binzel, R.P., Bus, S.J., Burbine, T.H., MothDiniz, T., Angeli, C.A., Florczak, M., \& Harris, A.W. 2000, Science 288, 2033

Marzari, F., Cellino, A., Davis, D.R., Farinella, P., Zappalá, V., \& Vanzani, V. 1996, Astron. Astrophys. 316, 1996

McCord, T.B., Adams, J.B., \& Johnson, T.V. 1970, Science 168, 1445

Michtchenko, T.A., Lazzaro, D., Ferraz-Mello, S., \& Roig, F. 2002, Icarus 158, 343

Mittlefehldt, D.W., McCoy, T.J., Goodrich, C.A., \& Kracher, A. 1988, in: J.J. Papike (ed.), Planetary Materials (Mineralogical Society of America) 36, 4

Mittlefehldt, D.W. 2005, Meteoritics. \& Planet. Sci. in press

Nyquist, L., Takeda, H., Shih, C.-Y., \& Wiesmann, H. 2004, Lunar \& Planet. Sci XXXV \#1330

Pieters, C.M. \& Binzel, R.P. 1994, Lunar E Planetary Science Conference XXV, 1083.

Pieters, C.M., Taylor, L.A., Noble, S.K., Keller, L.P., Hapke, B., Morris, R.V., Allen, C.C., McKay, D.S., \& Wentworth, S. 2000, Meteoritics \& Planetary Science 35, 1101

Ryder, G. 2002, Journal Geophysical Research 107 (E4, 10.1029/2001JE001583)

Rivkin, A.S., McFadden, L.A., Binzel, R.P., \& Sykes, M. 2005, Icarus in press

Russell, C.T., Coradini, A., Christensen, U., De Sanctis, M.C., Feldman, W.C., Jaumann, R., Keller, H.U., Konopliv, A., McCord, T.B., McFadden, L.A., McSween Jr., H.Y., Mottola, S., Neukum, G., Pieters, C.M., Prettyman, T.H., Raymond, C.A., Smith, D.E., Sykes, M.V., Williams, B.G., Wise, J., \& Zuber, M.T. 2004, Planetary 83 Space Science 52, 465

Sasaki, S., Hiroi, T., Nakamura, K., Hamabe, Y., Kurahashi, E., \& Yamada-J, M. 2002, Adv. Space Rev. 29 (5), 783

Takeda, H., Mori, H., Delaney, J.S., Prinz, M., Harlow, G.E., \& Ishi, T. 1983, Mem. Natl. Inst. Polar Res. Spec. 30, 181

Takeda, H. 1997, Meteoritics $\&$ Planet. Sci. 32, 841

Treiman, A.H., Lanzirotti, A., \& Xirouchakis, D. 2004, Earth 83 Planetary Science Letters 219, 189

Thomas, P.C., Binzel, R.P., Gaffey, M.J., Storrs, A.D., Wells, E.N., \& Zellner, B.H. 1997, Science 277,1492

Vernazza, P., Mothé-Diniz, T., Barucci, M.A., Birlan, M., Carvano, J.M., Strazzulla, G., Fulchignoni, M., \& Migliorini, A. 2005, Astron. Astrophy. 436, 1113

Wiechert, U., Halliday, A.N., Lee, D.-C., Snyder, G.A., Taylor, L.A., \& Rumble, D. 2001, Science-Verificar 294, 345

Wiechert, U.H., Halliday, A.N., Palme, H., \& Rumble, D. 2004, Earth \& Planetary Science Letters 221, 373

Wisdom, J. 1985, Nature 315, 731

Yamaguchi, A., Clayton, R.N., Mayeda, T.K., Ebihara, M., Oura, Y., Miura, Y.N., Haramura, H., Misawa, K., Kojima, H., \& Nagao, K. 2002, Science 296, 334 